\title{
Prevalence of Traumatic Brain Injury Among Trauma Patients in Ethiopia: Systematic Review and Meta-Analysis
}

\author{
Semagn Mekonnen Abate, ${ }^{1}$ Bedru Jemal Abafita, ${ }^{1}$ Tesfanew Bekele ${ }^{2}$ \\ ${ }^{1}$ Department of Anesthesiology, College of Health Sciences and Medicine, Dilla University, Dilla \\ Ethiopia \\ ${ }^{2}$ Department of Surgery, College of Health Sciences and Medicine, Dilla University, Dilla Ethiopia
}

Correspondence to: Dr. Semagn Mekonnen Abate; email: semmek17@gmail.com

Received: 06 December 2019; Revised: 29 February 2020; Accepted: 25 April 2020; Available online: 21 June 2020

\begin{abstract}
Background: Traumatic brain injury (TBI) is a TBI among trauma patients in Ethiopia when compared common cause of mortality and disability in young age populations, particularly in children and adolescents. The objective of this systematic review and metaanalysis was to assess the prevalence of TBI among trauma patients in Ethiopia. Methods: A three-stage search strategy was conducted on PubMed/Medline, Science Direct and African Journals Online, and a grey literature search was conducted on Google Scholar. Data were analyzed with $\mathrm{R}$ version 3.6.1. Results: The pooled prevalence of TBI in Ethiopia was 20\% (95\% confidence interval (CI), 11-32). Subgroup analysis revealed that road traffic accident was the commonest mechanism of injury in Ethiopia at 21\% (95\% IC, 15$30)$, next was assault at $18 \%$ (95\% CI, 5-48). Conclusion: This review shows a high prevalence of with continental and global reports. Policymakers and stakeholders should work on regulatory laws on transportation; pre-hospital emergency medical care system is also in high demand.

\section{Protocol registration: CRD42020146643.}

Keywords: Head injury, Road traffic accident, Assault, Ethiopia

Ann Afr Surg. 2021;18(1):10-17

DOI: http://dx.doi.org/10.4314/aas.v18i1.3

Conflicts of Interest: None

Funding: None

(C) 2021 Author. This work is licensed under the Creative Commons Attribution 4.0 International License.
\end{abstract}

\section{Introduction}

Head injury is defined as any trauma to the head resulting from external mechanical force, such as rapid acceleration or deceleration, impact or penetration (1). The Centers for Disease Control and Prevention defines traumatic brain injury (TBI) as a disruption in the normal function of the brain that can be caused by a bump, blow, or jolt to the head, or a penetrating head injury (2). Head injury is a common cause of mortality and disability in the young age population, particularly in children and adolescents (1). Among all trauma-related injuries, head injury is considered the greatest contributor to death and

disability globally (3). In addition, besides the impact on the individual, TBI can negatively affect families, communities, and the economy of the country through loss of productive age groups and treatment costs (4). It is estimated that in the USA about 5.3 million people are living with a TBI-related disability (5), and 7.7 million people in the European Union who have experienced TBI have disabilities (6). The World Health Organization (WHO) estimates that each year more than 10 million people in the world sustain TBI, resulting in death or severe disability (7). 
The report on the Global burden of disease study shows there were 27.08 million new cases of TBI in 2016. The report also revealed that TBI caused 8.1 million years of life lived with disability. Sub-Saharan Africa had 2.9 million new cases of TBI, equivalent to 360 per 100,000 population. The report also showed that Ethiopia accounted for 659 (624-703) TBI cases per 100,000 population, roughly representing $10.8 \%$ of Sub-Saharan Africa and $28 \%$ of eastern Sub-Saharan Africa TBI cases (3).

The incidence of TBI globally is rising, owing mainly to injuries associated with increases in population density and increased use of motor vehicles, particularly in middle- and low-income countries (8). Globally, road traffic accidents are responsible for more than 50 million injuries every year, with about 1.2 million ending in death $(3,9,10)$. Indeed, many TBIs are the result of motor-related accidents, but the pattern of injury varies across regions: in high-income countries, individuals with TBI are generally motor vehicle occupants, whereas in middle- and low-income countries patients with TBI are often vulnerable road-traffic users such as pedestrians, cyclists, and motorcyclists. Increased motorization combined with inadequate traffic education and slow implementation of traffic safety regulations are the main causes of the increasing incidence of TBI in low- and middle-income countries (11).

The prevalence of TBI among trauma patients in Ethiopia varied from town to town. A study conducted at Addis Ababa Emergency Center, Tikur Anbessa specialized hospital, showed that among 204 head injury patients enrolled in the study, $41 \%$ of injuries occurred from a road traffic accident and 21 patients died (10.3\%) (12). Another study in Myungsun Christian Medical Center had a $24.9 \%$ prevalence of head injury among trauma patients visiting the adult emergency department (ED): road traffic accident was the leading cause of head injury (43.5\%), next was fall accident $35.8 \%$ (13). A study done at Jimma University Teaching Hospital showed prevalence of head injury of $3.69 \%$ among patients admitted to adult ED, interpersonal violence accounted for $54.1 \%$ and road traffic accidents $31.1 \%$ (14). Another study in Tikur Anbessa specialized hospital shows a $1.9 \%$ mortality rate out of 9,956 patients who visited the ED; the most common cause of death was head injury (21.5\%) (15). A study conducted at Ayder Referral Hospital shows the prevalence of head injury from a review of records of road traffic accident was $48.7 \%$ (16).

Another study conducted at Dilla University referral hospital shows the prevalence of head injury was $32.1 \%$, and road traffic accident was the main cause of head injury (17). A study at Gondar University referral hospital also showed a prevalence of $40.5 \%$ for head injury; of these $46.7 \%$ occurred due to interpersonal fight (18). A study in Ayder referral hospital showed prevalence of head injury was $56.3 \%$ and the most common causes were fall accident $(41.9 \%)$, road traffic accident $(24.9 \%)$, and interpersonal violence $(24.8 \%)$ (19). In Ethiopia, a national prevalence of head injury is lacking, and health planners and policymakers use demographic health surveys. The current study aims to assess the national prevalence of TBI among trauma patients in Ethiopia.

\section{Methods}

\section{Protocol and registration}

The systematic review and meta-analysis was conducted based on the Preferred Reporting Items for Systematic and Meta-analysis (PRISMA) protocols. This systematic review and meta-analysis was registered in Prospero international prospective register of systematic reviews (PROSPERO identifier CRD42020146643)

\section{Eligibility criteria}

Types of studies

All cross-sectional studies reporting the prevalence of traumatic brain injury among trauma patients in Ethiopia, without any date and language restrictions, were incorporated.

\section{Types of participants}

Participants were all trauma cases having a head and/or neck injury.

Outcomes of interest

The primary outcome of interest was the prevalence of TBI among trauma patients in Ethiopia. Risk factors for TBI among trauma patients in different regions of the country were secondary outcomes. 


\section{Context}

The review incorporates studies conducted in Ethiopia. Inclusion criteria

The review included all cross-sectional studies to assess the magnitude of TBI, and other cross-sectional studies conducted on pattern and outcomes of trauma that report TBI as a secondary outcome.

\section{Exclusion criteria}

Studies that didn't report TBI, randomized controlled trials, cohort and case-control studies, systemic reviews, and case reports and series were excluded.

\section{Search strategy}

The search strategy intended to explore all available published and unpublished studies on traumatic brain injury among trauma patients in Ethiopia without date and language restrictions. A three-step search strategy was employed in this review. An initial search on PubMed/Medline, Science Direct and African Journals Online was carried out, followed by an analysis of the text words contained in the Title/Abstract and indexed terms. A second search combined free text words and indexed terms with Boolean operators. The third search used reference lists of all identified reports and articles for additional studies. Finally, an additional and grey literature search was conducted on Google Scholar up to 10 pages. Supplementary material 1 shows the search strategy conducted in PubMed.

\section{Data extraction}

Data from each study were extracted with a customized format by two independent authors. Disagreements between the two independent authors were resolved by the other two authors. The extracted data include author names, date of publication, sample size, prevalence of head injury among trauma patients, mean age of participants, and risk factors for the trauma. Finally, data were imported for analysis in $\mathrm{R}$ version 3.6.1.

\section{Data analysis}

Data analysis was carried out in $\mathrm{R}$ version 3.6.1 with meta and metafor packages. The pooled prevalence of TBI was determined with a random effect model as there was substantial heterogeneity. The heterogeneity among the included studies was checked with forest plot, $\chi 2$ test, I2 test, and the p-values. Substantial heterogeneity among the included studies was investigated with subgroup analysis. Publication bias was checked with a funnel plot and the objective diagnostic test was conducted with Egger's correlation, Begg's regression tests, and trim and fill method. In addition, moderator analysis was carried out to identify the independent predictors of TBI among trauma patients. The results were presented based on the Preferred Reporting Items for Systemic Reviews and Meta-Analysis (PRISMA) (31).

\section{Results}

\section{Description of included studies}

A total of 198 articles were identified from different databases, as described in the methodology section with the PRISMA flow diagram (Figure 1). Twenty-one articles were selected for evaluation after successive screening. Twelve articles with 14,017 participants reporting the prevalence of head injury as a primary outcome were included and the characteristics of each included study were described (Table 1), and the rest were excluded with reasons (Supplementary material 1). The included studies were published from 2011 to 2019; sample size ranged from 171 to 4,153 . The mean (SD) ages of the included studies varied from $24.7 \pm 15.8$ to $30.7 \pm 14$ years.

Nine studies were conducted in the Oromia region (3), Addis Ababa (3) and SNNPR (3), two in Amhara and one in Tigray regional states. However, no study reported the magnitude of TBI in Somalia, Afar, Gambella, Benshangul Gumuz, and Dire Dawa City administrations. The five included studies assessed the prevalence and outcomes of TBI; the remaining seven studies were on pattern and outcomes of trauma and they reported TBI. Most included studies reported the prevalence of TBI alone and the rest reported the prevalence of head and neck injury. Two of the included studies used total admission to calculate prevalence and 10 of the included studies used the total trauma cases as a source of population. Seven studies identified road traffic accident as the main mechanism of TBI, followed 
by assault; five studies reported assault as the main mechanism of injury, next was road traffic accident.

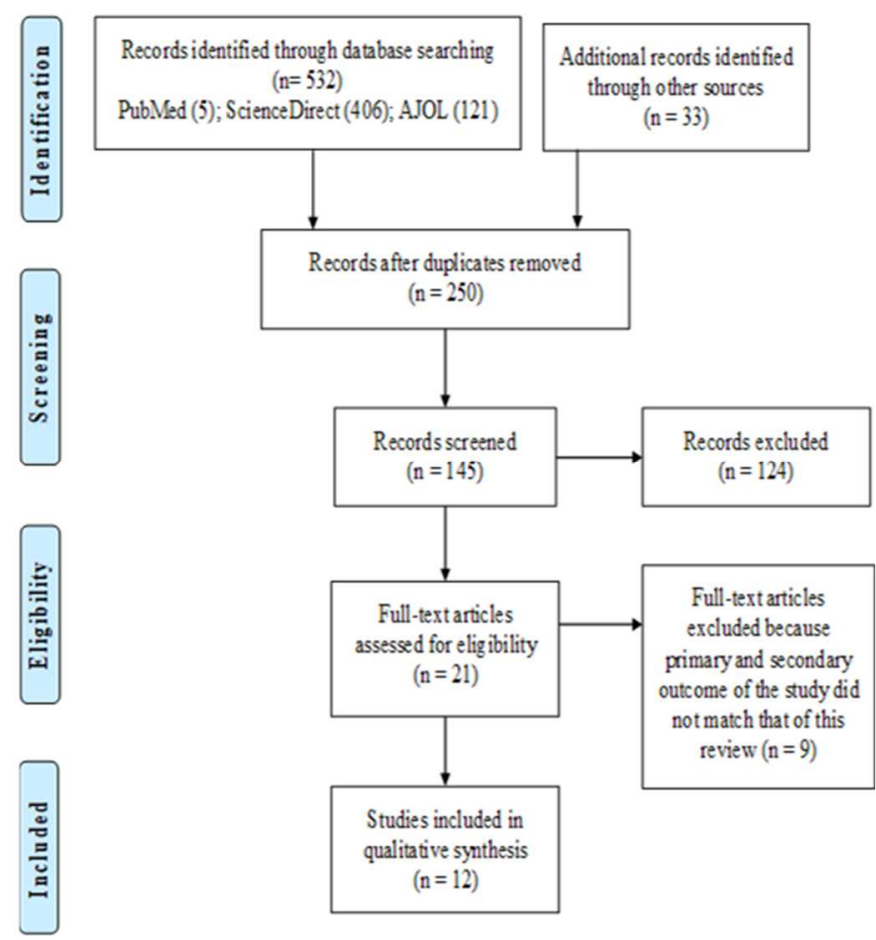

Figure 1: PRISMA flow diagram for included studies

\section{Assessment of methodological quality}

Articles identified for retrieval were assessed for methodological quality by two independent authors, using a standardized critical appraisal tool adapted from the Joanna Briggs Institute, before inclusion in the review (20) (Table 2). Disagreements between the authors appraising the articles were resolved through discussion with the other two authors. Articles with average scores greater than $50 \%$ were included for data extraction.

\section{Meta-analysis}

Twelve studies reporting TBI were included for metaanalysis: five studies were on the prevalence of TBI, and seven were on patterns of trauma but were included in the meta-analysis because of the reported prevalence of TBI. The pooled prevalence of TBI in Ethiopia was 20\% (95\% CI, 11-32 studies, 12,915 participants) (Figure 2). Subgroup analysis by region showed prevalence of TBI was highest in Tigray state, $49 \%$ (95\% CI, 43-54, 1 study, 322 participants), next was Oromia state $20 \%$ (95\% CI, 3-66, 3 studies, 4972 participants); the lowest were in Amhara state 15\% (95\% CI, 3-53, 2 studies, 4413 participants), next were Addis Ababa and Sothern Nations Nationalities People's Region (Figure 3).

Subgroup analysis by mechanism of injury revealed road traffic accident was the highest mechanism of injury in Ethiopia at 21\% (95\% CI, 15-30, 7 studies, 3530 participants), next was assault at 18\% (95\% CI, 548, 5 studies, 9,385 participants) (Figure 4).

The funnel plot for evaluating publication bias did not show asymmetric funnel plot (Figure 5). Also, the rank correlation and Egger's regression test did not show a significant difference for small study effect ( $p$-value $>0.05$ ). Moderator analysis failed to identify the effects of study quality, mean age of participants, year of publication and sample size on effect estimate of TBI among trauma patients, $\mathrm{p}>0.05$.

\section{Discussion}

The pooled prevalence of TBI in Ethiopia was 20\% (95\% CI, 11-32). The finding of this review is in line with a systemic review conducted in the Sub-Saharan African region. The similarity may be explained by relatively similar socio-economic status, mode of transportation, and infrastructure within these regions (30).

The global report on traumatic brain injury revealed that TBI prevalence was very low in the USA, China, Norway, and Finland (3) than in this systemic review. The possible explanation for this discrepancy might be due to road safety protocols, better infrastructure, and community awareness towards possible risk factors for injury including violence, driving speed, substance abuse and use of safety belts (3). This systemic review revealed that personal violence/assault was the second most common cause of TBI in Ethiopia. 
Table 1: Description of included studies

\begin{tabular}{|c|c|c|c|c|c|c|c|c|}
\hline Author & Year & Event & Sample & $\begin{array}{l}\text { Source } \\
\text { population }\end{array}$ & Region & Injury type & $\begin{array}{l}\text { Injury } \\
\text { mechanism }\end{array}$ & Quality scores \\
\hline Ayele et al.(21) & 2017 & 174 & 4153 & all admission & Amhara & head & Assault & 8 \\
\hline Tesfaye et al.(16) & 2019 & 157 & 322 & trauma & Tigray & head & RTA & 8 \\
\hline Walle et al.(18) & 2018 & 104 & 260 & trauma & Amhara & head & Assault & 8 \\
\hline Bulto et al.(22) & 2018 & 164 & 220 & trauma & Oromia & head & Assault & 7 \\
\hline Semagn et al.(25) & 2019 & 44 & 376 & trauma & SNNPR & head & RTA & 7 \\
\hline Kifle et al.(23) & 2011 & 139 & 1102 & trauma & Oromia & head and neck & Assault & 6 \\
\hline Taddesse et al. (26) & 2014 & 140 & 989 & trauma & Addis Ababa & head and neck & RTA & 6 \\
\hline Tiruneh et al.(18) & 2014 & 57 & 356 & trauma & Addis Ababa & head and neck & RTA & 6 \\
\hline Eshete et al.(17) & 2018 & 125 & 643 & trauma & SNNPR & head & RTA & 5 \\
\hline Ketema et al.(14) & 2015 & 135 & 3650 & all admission & Oromia & head & Assault & 5 \\
\hline Negussie.(24) & 2018 & 45 & 171 & trauma & SNNPR & head and neck & RTA & 5 \\
\hline Wosen et al.(13) & 2017 & 168 & 673 & trauma & Addis Ababa & head & RTA & 5 \\
\hline
\end{tabular}

All study designs were cross-sectional

RTA $=$ Road traffic accidents

Table 2: Methodological quality of included studies

Abbreviations: Q: question; $Y$ : yes; $N$ : no

\begin{tabular}{llllllllll}
\hline Author & Q1 & Q2 & Q3 & Q4 & Q5 & Q6 & Q7 & Q8 & Score \\
\hline Ayele et al. (21) & Y & Y & Y & Y & Y & Y & Y & Y & 8 \\
Tesfay et al.(16) & Y & Y & Y & Y & Y & Y & Y & Y & 8 \\
Walle et al.(18) & Y & Y & Y & Y & Y & Y & Y & Y & 8 \\
Bulto et al.(22) & N & Y & Y & N & Y & Y & Y & Y & 7 \\
Semagn et al.(25) & Y & Y & Y & N & Y & Y & Y & Y & 7 \\
Kifle et al.(23) & N & Y & Y & N & Y & Y & Y & Y & 6 \\
Taddesse et al.(18) & N & Y & Y & N & Y & Y & Y & Y & 6 \\
Tiruneh et al.(26) & Y & Y & N & N & Y & Y & Y & Y & 6 \\
Eshete et al.(17) & N & Y & N & N & Y & Y & Y & Y & 5 \\
Ketema et al.(14) & Y & Y & Y & Y & N & N & Y & N & 5 \\
Negussie.(24) & Y & Y & Y & Y & N & N & Y & N & 5 \\
Wosen et al.(13) & Y & Y & Y & Y & N & N & Y & N & 5 \\
\hline
\end{tabular}

Personal violence was the least mentioned cause of TBI, as shown in other systemic reviews conducted in the Sub-Saharan region and globally $(3,28,30)$. This discrepancy might be due to the current tribalism and instability in the Ethiopian context.

The systemic review revealed that road traffic accident was the most common cause of TBI, which corroborates another systemic review conducted in the Sub-Saharan region (30). The similarity may be explained by the similar mode of transportation, socioeconomic status, transportation and principles of road safety (30).

The subgroup analysis showed a higher prevalence of TBI in Tigray regional state than in other regional states. This discrepancy might be due to few studies carried out, only one study on the prevalence of TBI in this region, study setting and small sample size. 

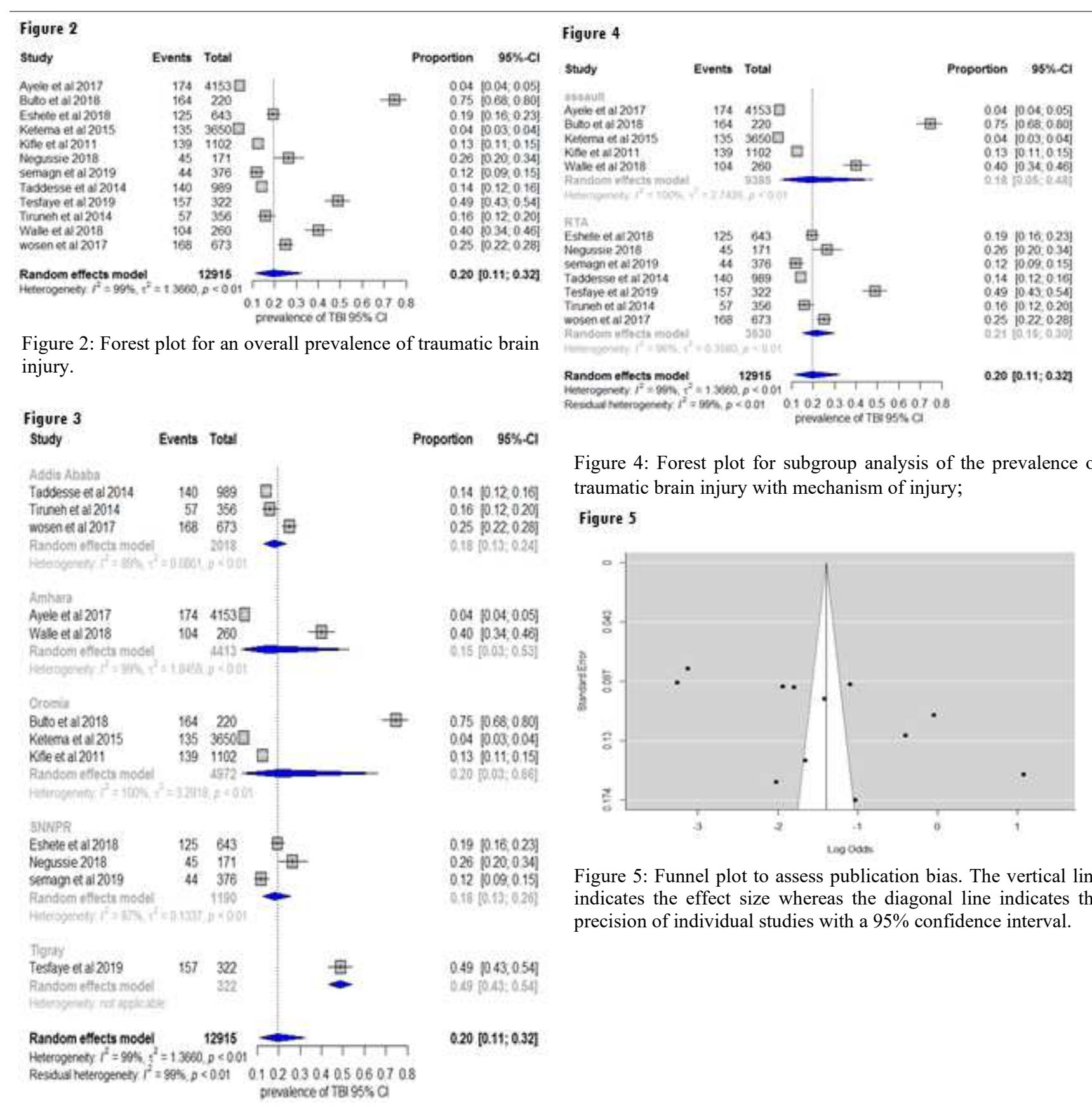

Figure 4: Forest plot for subgroup analysis of the prevalence of traumatic brain injury with mechanism of injury;

Figure 5

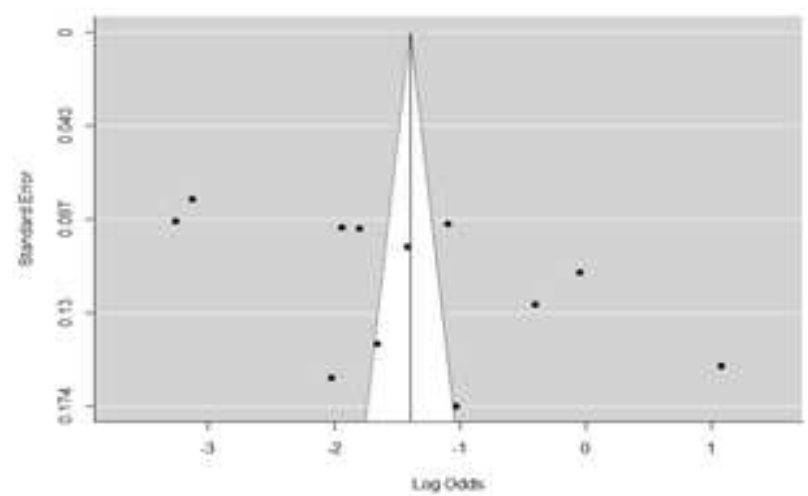

Figure 5: Funnel plot to assess publication bias. The vertical line indicates the effect size whereas the diagonal line indicates the precision of individual studies with a $95 \%$ confidence interval.

Figure 3: Forest plot for subgroup analysis of the prevalence of traumatic brain injury with the region;

\section{Limitation of the study}

The review incorporated a few studies assessing the prevalence of TBI among trauma patients. Besides, the number of studies was small in some regions and other regions had no studies at all assessing the prevalence of TBI. As a result, the review had substantial heterogeneity. The methodological qualities of the included studies were high to moderate, as shown with the methodological assessment tool. However, the overall quality of evidence was low because there was an inconsistency effect estimate within studies, and also because it is a meta-analysis of cross-sectional studies (low levels of evidence). 


\section{Conclusion}

The review revealed that the prevalence of TBI among trauma patients in Ethiopia is high when it is compared with continental and global reports. Road traffic accident and assault were the most common causes of TBI in Ethiopia. To reduce the prevalence of TBI in Ethiopia, policymakers and stakeholders should work on awareness-creation strategies on road safety, regulatory laws on transportation, mode of transport, types of vehicles, driving license, and pre-hospital emergency medical care system that is highly in demand. More evidence with a large sample size on the prevalence of TBI and associated factors from each region is still needed.

\section{References}

1. Rath G, Ray B. Head injury: assessment and early management. Pract Guidel Anesth. 2016;(September):53-53.

2. Defillo A. Letter to the Editor. J Neurosurg. 2011;116(1):2567.

3. James SL, Theadom A, Ellenbogen RG et al. Global, regional, and national burden of traumatic brain injury and spinal cord injury, 1990-2016: a systematic analysis for the Global Burden of Disease Study 2016. Lancet Neurol. 2019;18(1):56-87.

4. Lookstein RA, Giordano CF. Deep vein thrombosis: endovascular management. Medicine (Baltimore). 2010;28695 .

5. Langlois J, Rutland-Brown W. Traumatic brain injury in the United States: The Future of Registries and Data Systems. Centers Dis Control Prev Natl Cent Inj Prev Control. 2005; (June 2005).

6. Tagliaferri F, Compagnone C, Korsic M, et al. A systematic review of brain injury epidemiology in Europe. Acta Neurochir (Wien). 2006;148(3):255-67.

7. Hyder AA, Wunderlich CA, Puvanachandra P, Gururaj G, Kobusingye OC. The impact of traumatic brain injuries: A global perspective. NeuroRehabilitation. 2007;22(5):341-53.

8. Maas AIR, Stocchetti N, Bullock R. Moderate and severe traumatic brain injury in adults, Maas AIR et al. 2008;7(August).

9. Jacobs G, Aeron-Thomas A. A review of global road accident fatalities. RoSPA Road Saf Congr [Internet]. 1999;1-15. Available from: http://www.transport-links.org/transport_links /filearea/publications/1_771_Pa3568.pdf

10. World Health Organization (WHO). Summary World Report on Disability. World Health [Internet]. 2011;1-24. Available from: www.who.int/about/licensing/copyright form/en/index. html\%0Ahttp://www.larchetoronto.org/wordpress/wpcontent/uploads/2012/01/launch-of-World-Report-onDisability-Jan-27-121.pdf

11. Roozenbeek B, Maas AIR, Menon DK. Changing patterns in the epidemiology of traumatic brain injury. Nat Rev Neurol [Internet]. 2013;9(4):231-6. Available from: http://dx.doi.org/10.1038/nrneurol.2013.22

12. Landes M, Venugopal R, Berman S, Heffernan S, Maskalyk J, Azazh A. Epidemiology, clinical characteristics and outcomes of head injured patients in an Ethiopian emergency centre. African J Emerg Med. 2017;7(3):130-4.

13. Woldmeskel A. Assessment of Magnitude and Outcomes of Head Injury in Myungsung Christian Medical Center (Korea Hospital), Addis Ababa, Ethiopia (Doctoral dissertation, Addis Ababa University).

14. Indeshaw K. Pattern of head injury among patients presented to adult emergency department of Jimma University Teaching Hospital, Jimma, South West Ethiopia (Doctoral dissertation, Addis Ababa University).

15. Hunchak C, Teklu S, Meshkat N, et al. Patterns and predictors of early mortality among emergency department patients in Addis Ababa, Ethiopia Public Health. BMC Res Notes. 2015;8(1):1-9.

16. Tesfay K, Assefa M, Zenebe D et al. Road traffic injured patients with severe GCS and organ injury had a poor prognosis: A retrospective cohort study. BMC Public Health. 2019;19(1):1-8.

17. Eshete A, Taye F. Magnitude of severe head injury and its associated factors among head injury patients in Gedeo Zone, Southern Ethiopia: A two-year retrospective study. Ethiop J Health Sci. 2018;28(3):323-30.

18. Walle TA, Tiruneh BT, Bashah DT. Prevalence of head injury and associated factors among trauma patients visiting the surgical emergency department of Gondar University Referral Hospital, Northwest Ethiopia, 2016. A cross-sectional study. Int J Africa Nurs Sci [Internet]. 2018;9(March):57-61. Available from: https://doi.org/10.1016/j.ijans.2018.08.002

19. Amdeslasie F, Kidanu M, Tadesse A, et al. Head injury admissions at a referral hospital in Ethiopia. Ethiop Med J. 2017;55(3):195-202.

20. Moola S, Munn Z, Tufanaru C, Aromataris E, Sears K, Sfetcu $\mathrm{R}$, et al. Checklist for Analytical Cross-Sectional Studies. Joanna Briggs Inst Rev Man [Internet]. 2017;6. Available from: Http://joannabriggs.org/research/critical-appraisal-tools.html

21. Surgery OA. Magnitude and patterns of injuries among patients in Gondar University Hospital, northwest Ethiopia: an institutional-based study. 2017;25-31.

22. Bulto LN, Dessie Y, Geda B. Magnitude, causes and characteristics of trauma victims visiting emergency and surgical units of Dilchora Hospital, Eastern Ethiopia. Pan Afr Med J. 2018; 30:1-7.

23. Kifle W. Original article magnitude Ethiopia and pattern of injury in Jimma specialized hospital, southwest. 2004;(2).

24. Negussie A, Getie A, Manaye E, Tekle T. Prevalence and outcome of injury in patients visiting the emergency department of Yirgalem General Hospital, Southern Ethiopia. 2018;1-5.

25. Abate SM, Abafita BJ, Kassim HM, et al. Epidemiology and outcomes of injuries among trauma patients in Ethiopia: A 5years retrospective analysis. Research Square [pre-print]. DOI:10.21203/rs.2.14348/v1

26. Tiruneh BT, Dachew BA, Bifftu BB. Incidence of road traffic injury and associated factors among patients visiting the Emergency Department of Tikur Anbessa Specialized Teaching Hospital, Addis Ababa, Ethiopia. 2020;2014.

27. Eaton J, Hanif AB, Grudziak J, Charles A. Epidemiology, management, and functional outcomes of traumatic brain injury in Sub-Saharan Africa. World Neurosurg. 2017;108:650-5.

28. El-Gindi S, Abdel Azeem A, Mahdy M. El daño cerebral traumático en Los países en desarrollo. Rev española Neuropsychol. 2001;3(3):3-11.

29. Bock-Oruma A, Odatuwa-Omagbemi D, Ikubor J. Pattern of 


\section{ABATE ET AL.}

head injury in the Emergency Department of the Delta State University Teaching Hospital, Oghara, Nigeria. Br J Med Med Res. 2016;15(4):1-6.

30. Qureshi JS, Ohm R, Rajala H, Mabedi C, Sadr-Azodi O, Andrén-Sandberg Å, et al. Head injury triage in a sub-Saharan
African urban population. Int J Surg [Internet]. 2013;11(3):2659.

Available from: http://dx.doi.org/10.1016/j.ijsu.2013.01.011

31. Moher D, Liberati A, Tetzlaff J, Altman DG. PRISMA flow diagram PDF version. Ann Intern Med. 2014;151(4):264-9. 